\title{
AGC Tuning of an Inter-Connected Power System after Deregulation Using PSO
}

\author{
S. Rout ${ }^{1}$, K. Panda ${ }^{2}$ \\ Asst. Professor, Department of Electrical Engineering, C. V. Raman College of Engineering, Bhubaneswar, India ${ }^{1,2}$
}

\begin{abstract}
Automatic generation control (AGC) of an inter-connected power system provides power demand signals for AGC power generators to control frequency and tie-line power flow due to the large load changes or other disturbances. Occurrence of large megawatt imbalance causes large frequency deviations from its nominal value which may be a threat to secure operation of power system. It is important to suppress the disturbances by the coordination of governor systems .So, it is necessary to use secondary controller to increase the stability and performance of the AGC system. This paper provides an overview for the researcher of control strategies, as well as their current use in the field of AGC problem using Particle Swarm Optimization (PSO). The proposed PSO based controller is compared against conventional I-controller and the performance of the proposed controller is found better than the conventional method. Simulations have been performed using Matlab.
\end{abstract}

Keywords: AGC (Automatic Generation Control), Deregulated Power System, Particle Swarm Optimization (PSO), PID Controller, Area Control Error (ACE).

\section{INTRODUCTION}

Automatic generation control (AGC) maintains areas area interconnected power system has four principal generation changes due to sudden change in load objectives when operating in either the so-called normal or perturbations. The purpose of AGC is not only to maintain preventive operating states:

system frequency at nominal value but also to allocate - Ensuring zero steady state error for frequency generation between different areas at economical value and to keep the accurate value of tie line flows between different areas. The availability of an accurate model of the system is very crucial because it contains different uncertainties due to sudden change in load variation [1].

Currently, the electric power industry is in transition from vertically integrated utilities to an industry that will incorporate competitive companies, which is known as deregulation. Also, major changes have been introduced into the structure of electric power utilities all around the world. The reason for this was to improve the efficiency in the operation of power system by means of deregulating the industry and opening it up to private competition. In this new framework, consumers will have an opportunity to make a choice among competing providers of electric energy.

The net effect of such changes will mean that the transmission generation and distribution systems must now adapt to a new set of rules dictated by open markets. The deregulated power system consists of GENCOs, TRANSCOs and DISCOs with an open access policy. In the new structure, GENCOs may or may not particulate in their own or other areas. Thus, various combinations of possible contracted scenarios between DISCOS and GENCOS are possible. All the transactions have to be cleared by the independent system operator or other risible organizations. Due to these, a study on simulation and optimization in an AGC system after deregulation [6],[7],[8],[9] This increases the complexity of the load frequency issue and calls for more insight and research. So here, the effect of bilateral contracts on the dynamics of the system is taken into account and the concept of DISCO participation matrix for these bilateral contracts is simulated. Automatic generation control (AGC) in a multideviations.

- Minimizing unscheduled tie line power flows between neighboring control areas.

- Getting good tracking for load demands and disturbances.

- Maintaining acceptable overshoot and settling time on the frequency and tie line power deviations.

Particle Swarm Optimization (PSO) algorithms are adopted in order to obtain the optimal parameters of the load-frequency controllers. Here, we adopt the PSO method because, PSO is easier to implement and there are fewer parameters to adjust than conventional method.

\section{A. Two Area Deregulated Power System:}

In the competitive environment of power system, the vertically integrated utility (VIU) no longer exists. Deregulated system will consist of GENCOs, DISCOs, and transmission companies (TRANSCOs) and independent system operator (ISO) [2]. However, the common AGC goals, i.e. restoring the frequency and the net interchanges to their desired values for each control area, still remain. The power system is assumed to contain two areas and each area includes two GENCOs and also two DISCOs as shown in Fig.1.

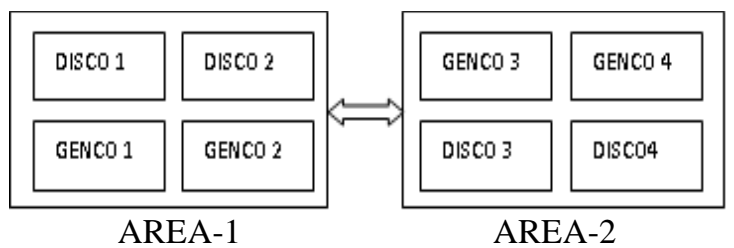

Fig. 1. Schematic diagram of a two-area system in deregulated power system 


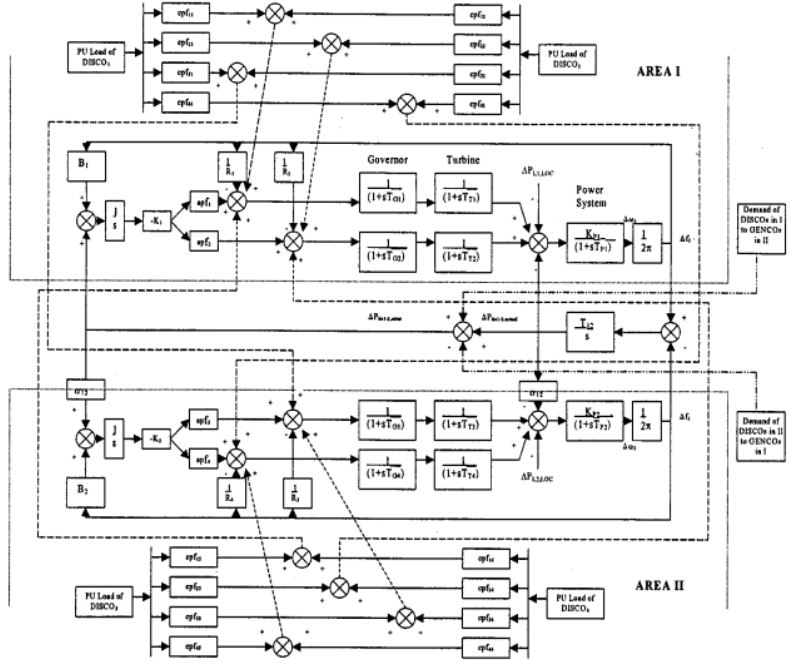

Fig. 2. Two-area AGC system block diagram in deregulated power system

The corresponding DPM will become:

$$
\left[\begin{array}{llll}
c p f_{11} & c p f_{12} & c p f_{13} & c p f_{14} \\
c p f_{21} & c p f_{22} & c p f_{23} & c p f_{24} \\
c p f_{31} & c p f_{32} & c p f_{33} & c p f_{34} \\
c p f_{41} & c p f_{42} & c p f_{43} & c p f_{44}
\end{array}\right]
$$

where cpf refers to "contract participation factor." Suppose that DISCO3 demands 0.1 pu MW power, out of which 0.025 puMW is demanded from GENCO1, 0.03 puMW from GENCO2, 0.035 puMW from GENCO3and 0.01 puMWfrom GENCO4.

Then column 3 entries in (1) are easily defined as:

$$
\begin{aligned}
& c p f_{13}=\frac{0.025}{0.1}=0.25, \text { cpf }_{23}=\frac{0.03}{0.1}=0.3 \\
& c p f_{33}=\frac{0.035}{0.1}=0.35, c p f_{43}=\frac{0.01}{0.1}=0.1
\end{aligned}
$$

Whenever a load demanded by a DISCO changes, it is reflected as a local load in the area to which this DISCO belongs. This corresponds to the local loads $\triangle P L 1$ and $\triangle P L 2$ and should be reflected in the deregulated AGC system block diagram at the point of input to the power system block.

In AGC control area is not only interconnected with one tie-line to one neighboring area but also with several tie-lines to neighboring control areas [3]. Tie-line power data are compared with the predetermined power, and the change in power is added with the biased frequency which is called area control error (ACE).

The ACE of each area is linear combination of biased frequency and tie-line error. This is the objective function taken for analysis in this paper.

$$
\begin{aligned}
& I T A E=\int_{0}^{t}\left(\Delta p_{\text {tie }}+\Delta f i\right)^{2} t d t \\
& (i=1,2 \ldots \ldots \ldots . . D) \text { (Where } \mathrm{D} \text { is the dimension) }
\end{aligned}
$$

\section{Technical Work Preparation}

\section{A .Particle Swarm Optimization (PSO): (PSO based PID controller)}

PSO is the search method to improve the speed of convergence and find the global optimum value of fitness function. It starts with a population of random solutions "particles" in a D-dimension space. The ith particle is represented by $X i=(x i 1, x i 2, \ldots, x i D)$. Each particle keeps track of its coordinates in hyperspace, which are associated with the fittest solution it has achieved so far. The value of the fitness for particle $i$ (pbest) is also stored as $P i=(p i 1, p i 2, \ldots, p i D)$.

The global version of the PSO keeps track of the overall best value (gbest), and its location, obtained thus far by any particle in the population. PSO consists of, at each step, changing the velocity of each particle toward its pbest and gbest according to Eq. (2). The velocity of particle $i$ is represented as $V i=(v i 1, v i 2$. . viD). Acceleration is weighted by a random term, with separate random numbers being generated for acceleration toward pbest and gbest. The position of the $i$ th particle is then updated according to Eq. (3) [4]

$$
\begin{aligned}
& v_{i d}=w \times v_{i d}+c_{1} \times \operatorname{rand}() \times\left(p_{i d}-x_{i d}\right)+c_{2} \\
& \times \operatorname{rand}() \times\left(p_{g d}-x_{i d}\right) \\
& x_{i d}=x_{i d}+c v_{i d}
\end{aligned}
$$

Where, Pid and Pgd are pbest and gbest. Several modifications have been proposed in the literature to improve the PSO algorithm speed and convergence toward the global minimum. One modification is to introduce a local-oriented paradigm (lbest) with different neighborhoods. It is concluded that gbest version performs best in terms of median number of iterations to converge.

However, Pbest version with neighborhoods of two is most resistant to local minima. PSO algorithm is further improved via using a time decreasing inertia weight, which leads to a reduction in the number of iterations [5].

Figure 3 shows the flowchart of the proposed PSO algorithm. This new approach features many advantages; it is simple, fast and easy to be coded. Also, its memory storage requirement is minimal. Moreover, this approach is advantageous over evolutionary and genetic algorithms in many ways.

First, PSO has memory. That is, every particle remembers its best solution (local best) as well as the group best solution (global best). Another advantage of PSO is that the initial population of the PSO is maintained, and so there is no need for applying operators to the population, a process that is time and memory-storage-consuming.

In addition, PSO is based on "constructive cooperation" between particles, in contrast with the genetic algorithms, which are based on "the survival of the fittest". 


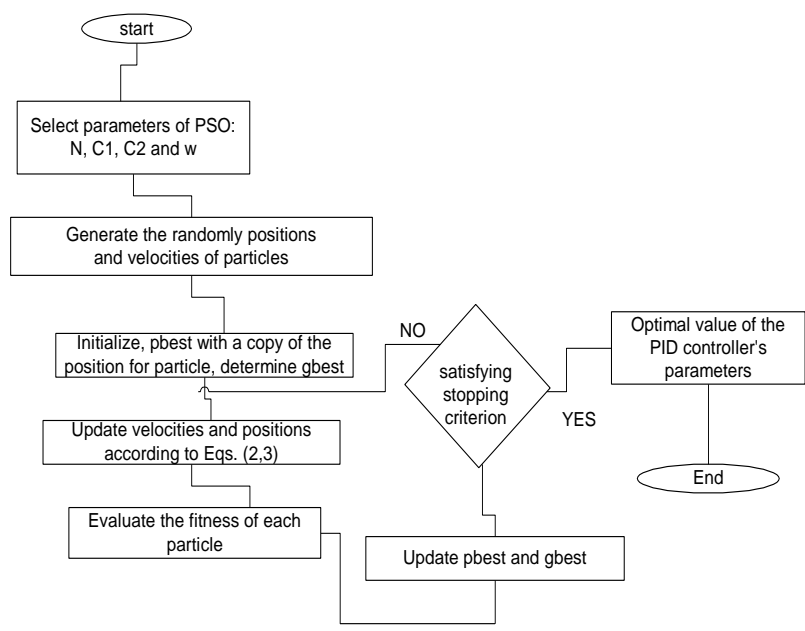

Fig3. Flowchart of the proposed PSO algorithm

\section{CASE STUDY}

A. Case 1: Base Case: Consider a case where the GENCOs in each areas participate equally in AGC; i.e., ACE participation factors are apf $1=0.5$,apf $2=1-\operatorname{apf} 1=0.5$ and similarly apf $3=$ apf $4=0.5$. Assume that load change only occurs in area I. Thus the load is demanded by only DISCO and DISCO . Let the value of this load demand be 0.1 pu MW for each of them.

The DPM matrix will be as follows:

$$
\left[\begin{array}{cccc}
0.5 & 0.5 & 0 & 0 \\
0.5 & 0.5 & 0 & 0 \\
0 & 0 & 0 & 0 \\
0 & 0 & 0 & 0
\end{array}\right]
$$

B. Case 2: Considering a case where all the DISCOs contract with the GENCOs for power as per the following DPM entries :

$$
\left[\begin{array}{cccc}
0.5 & 0.25 & 0 & 0.3 \\
0.2 & 0.25 & 0 & 0 \\
0 & 0.25 & 1 & 0.7 \\
0.3 & 0.25 & 0 & 0
\end{array}\right]
$$

It is assumed that each DISCO demands 0.1 pu MWpower from GENCOs as defined by cpfs in DPM matrix and each GENCO participates in AGC as defined by following apfs: apf $1=0.75$, apf $2=1-a p f 1=0.25$, apf $3=a p f 4=0.5$.

ACE participation factors affect only the transient behavior of the system and not the steady state behavior when uncontracted loads are absent.

\section{Case 3: Contract Violation}

It may happen that a DISCO violates a contract by demanding more power than that specified in the contract. This excess power is not contracted out to any GENCO. This uncontracted power must be supplied by the GENCOs in the same area as the DISCO. It must be reflected as a local load of the area but not as the contract demand. Consider case 2 again with a modification that DISCO demands 0.1 pu MW of excess power.
The total local load in area $\mathrm{I}(\Delta \mathrm{pL} 1, \mathrm{LOC})=$ Load of DISCO1+ load of DISCO2 $=(0.1+0.1)+0.1 \mathrm{pu} \mathrm{MW}=0.3 \mathrm{pu}$ MW. Similarly, the total local load in area $\mathrm{II}(\Delta \mathrm{pL} 2, \mathrm{LOC})=$ Load of DISCO3+ load of DISCO4=0.2 pu MW (no uncontracted load)

\section{SIMULATION RESULTS}

From the following simulation results, we found that the over-shoot, under-shoot and settling time are remarkably reduced adapting the PSO method. For PSO method, the values of the parameters are taken as follows:

$$
c_{1}=0.12, c_{2}=1.2, w=0.9, D=50
$$

For Base case, these are the following simulation results for frequency deviations of two areas and tie-line power and the power generated by the 4 GENCOS is shown in Fig 4 (a,b,c,d,e,f,g) respectively. For PSO-based Icontroller the integral constants for the 2areas are $K_{I 1}=0.0109, K_{I 2}=0.0124$ respectively.

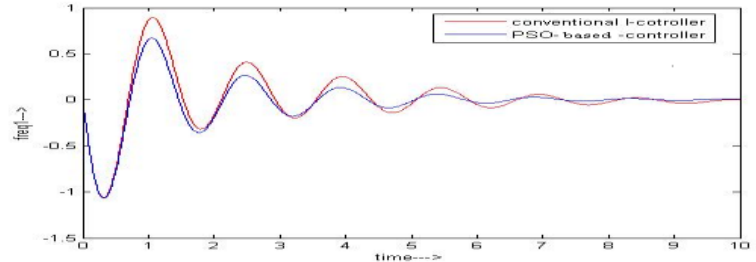

Fig.4a frequency deviation of area-1 with time

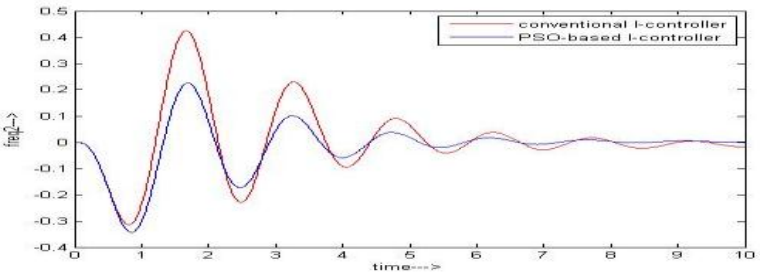

Fig.4b frequency deviation of area-2 with time

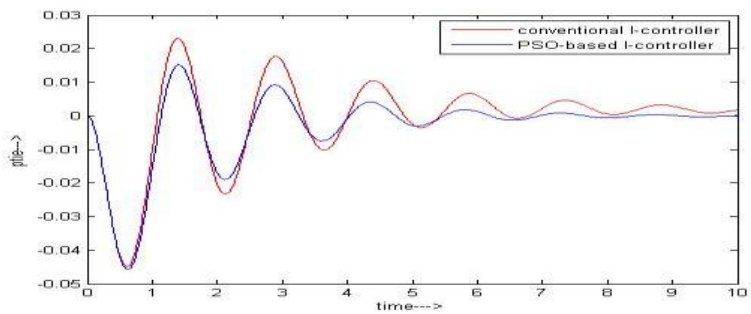

Fig.4c Tie-line power with time

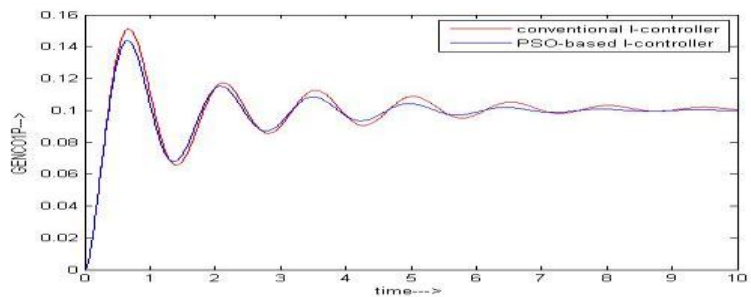

Fig.4d. Power generated by GENCO1 with time

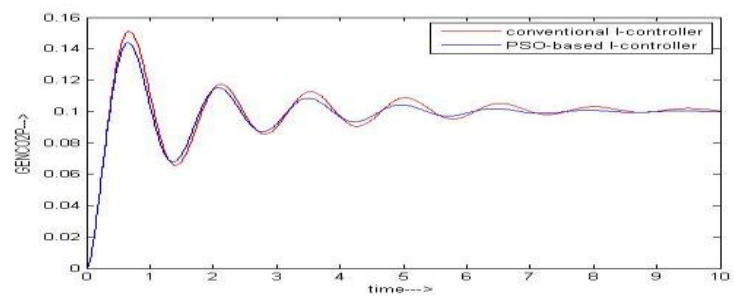

Fig.4e. Power generated by GENCO2 with time 
INTERNATIONAL JOURNAL OF INNOVATIVE RESEARCH IN ELECTRICAL, ELECTRONICS, INSTRUMENTATION AND CONTROL ENGINEERING

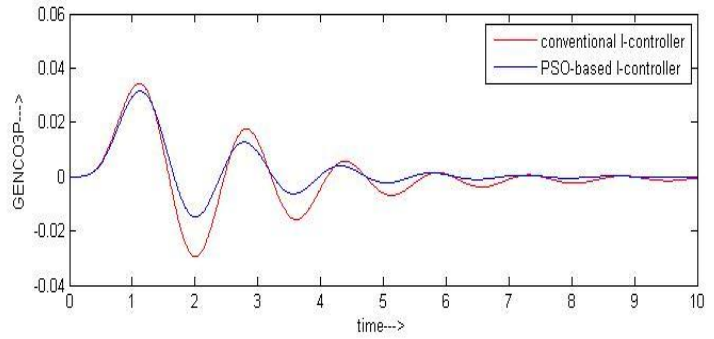

Fig.4f. Power generated by GENCO3 with time

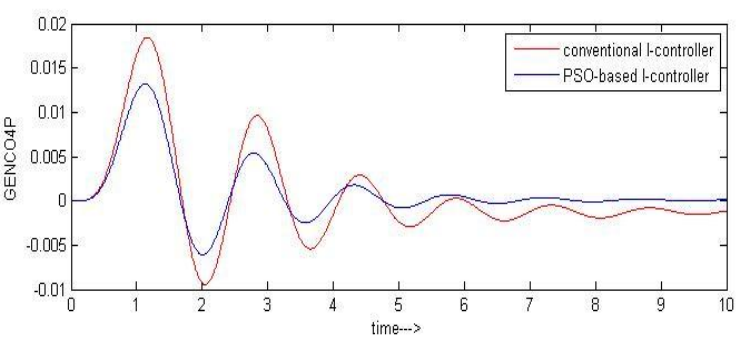

Fig.4g. Power generated by GENCO4 with time

The simulation results for second case are as follows: For $2^{\text {nd }}$ case in PSO-based I-controller, $K_{I 1}=0.0139, K_{I 2}=0.0126$ for the two areas respectively.

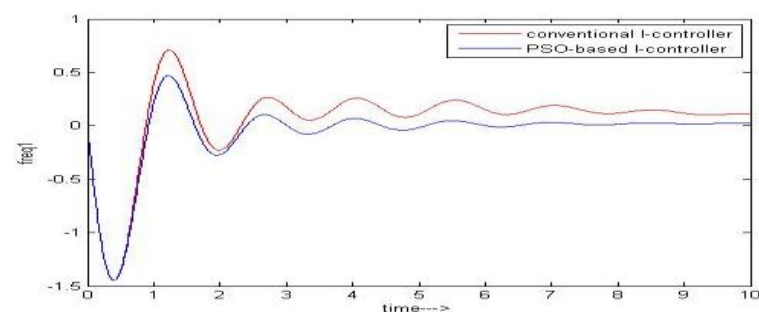

Fig.5a. Frequency deviation of area1 with time

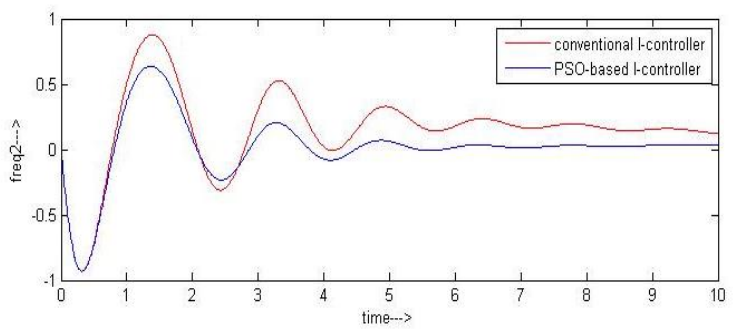

Fig.5b. Frequency deviation of area 2 with time

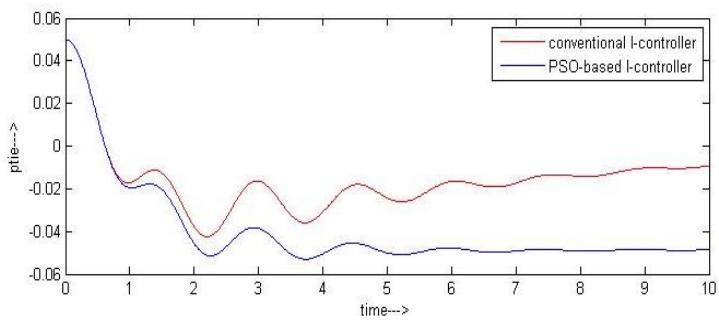

Fig.5c. Tie-line power with time

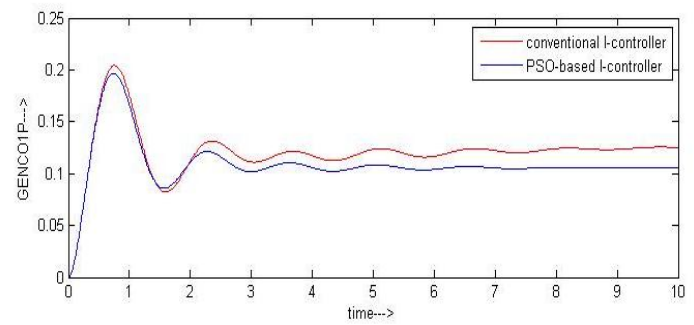

Fig.5d. Power generated by GENCO1 with time

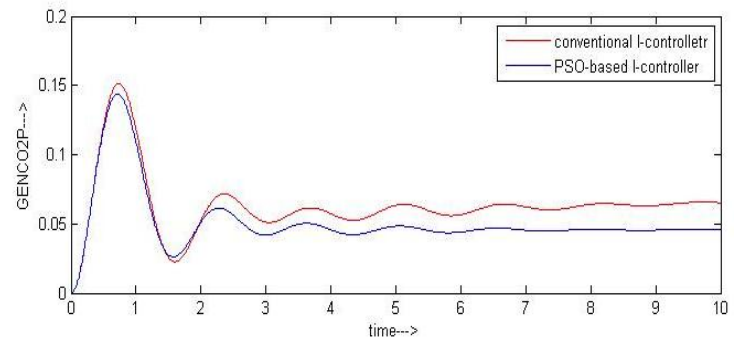

Fig.5e. Power generated by GENCO2 with time

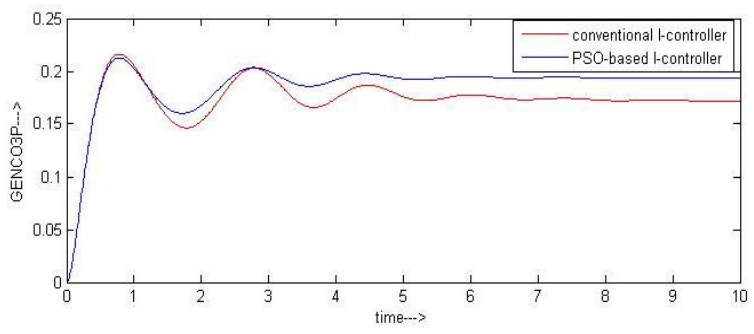

Fig.5f. Power generated by GENCO3 with time

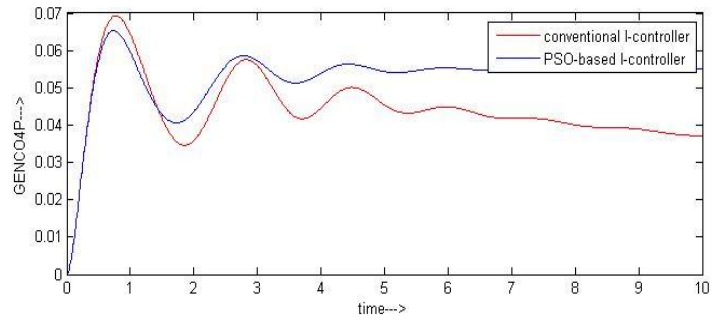

Fig.5f. Power generated by GENCO4 with time

The simulation results for third case are as follows: For $3^{\text {rd }}$ case, in PSO-based I-controller, $K_{I 1}=0.4388, K_{I 2}=0.4843$ for the two areas respectively.

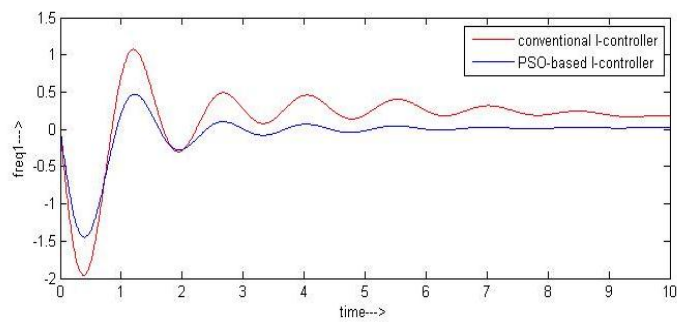

Fig.6a. Frequency deviation of area 1 with time

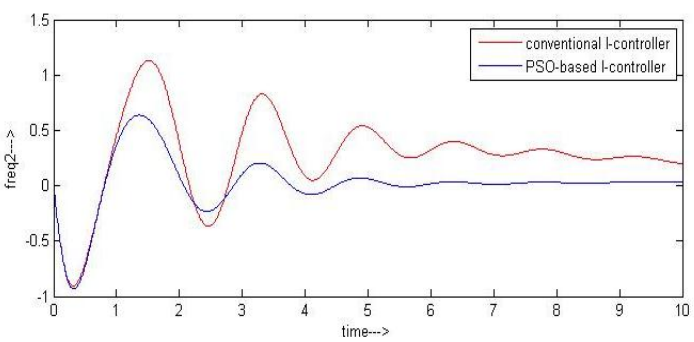

Fig.6b. Frequency deviation of area 2 with time

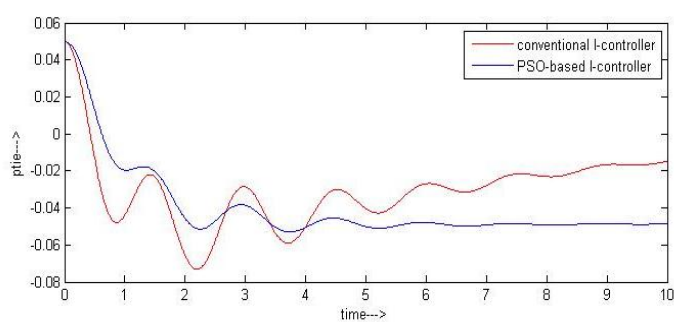

Fig.6c. Tie-line power with time

DOI 10.17148/IJIREEICE.2015.3810 


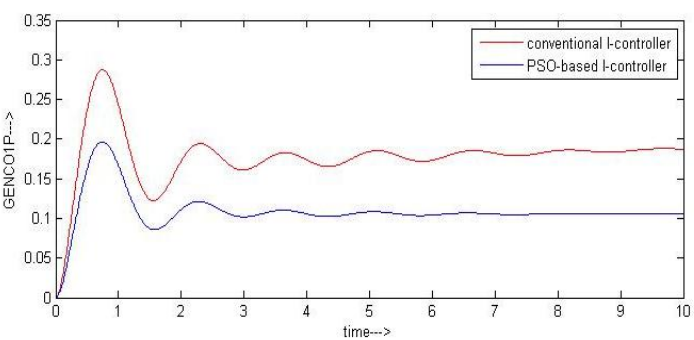

Fig.6d. Power generated by GENCO1 with time

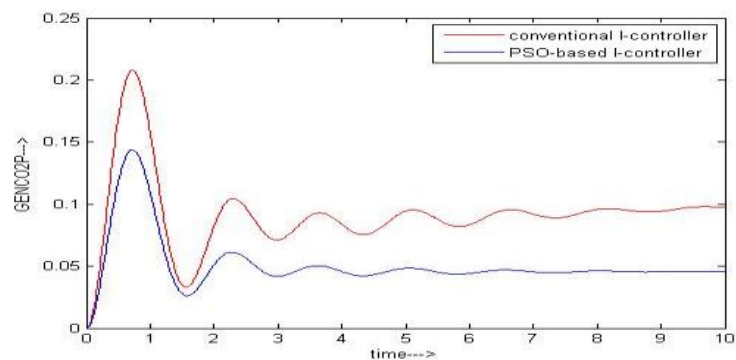

Fig.6e. Power generated by GENCO2 with time

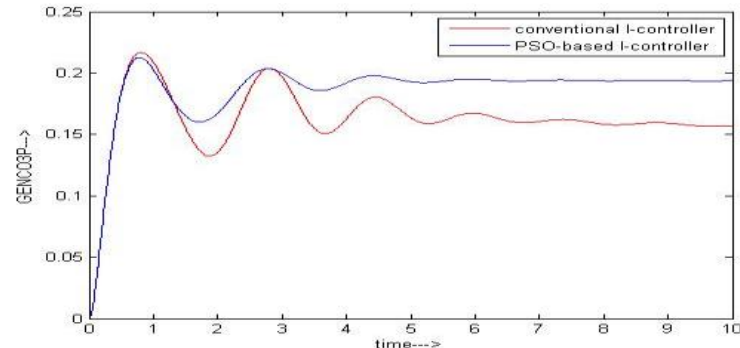

Fig.6f. Power generated by GENCO3 with time

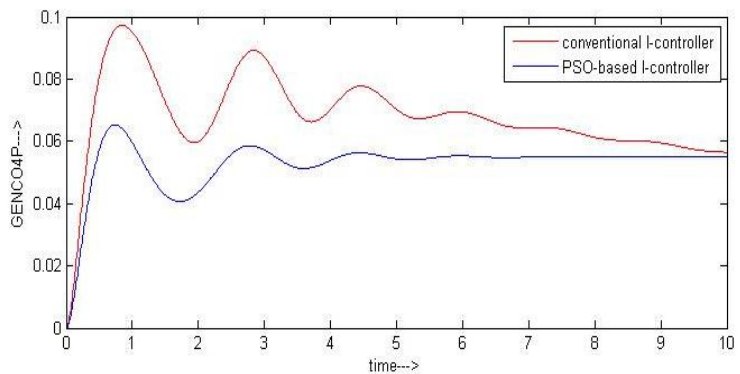

Fig.6g. Power generated by GENCO4 with time

\section{V.CONCLUSION}

The generalized AGC scheme model is considered here. LFC problem has been formulated as an optimization problem. Bilateral contracts can exist between DISCOs in one control area and GENCOs in other control areas. The use of a "DISCO Participation Matrix" facilitates the simulation of bilateral contracts. The PSO is algorithm proposed in this paper is easy to implement without additional computational complexity. Thereby experiments this algorithm gives quite promising results.

The effectiveness of the proposed strategy was tested on a two-area power system under possible contracts. The simulation results show that the proposed method is superior than the conventional method. The dynamic performance of system such as frequency and tie-lines power flows oscillations is significantly suppressed.

\section{APPENDIX}

$T_{G 1}=T_{G 2}=T_{G 3}=T_{G 4}=0.08 s, T_{T 1}=T_{T 2}=T_{T 3}=T_{T 4}=0.3 \mathrm{~s}$.

$K_{P 1}=K_{P 2}=120$

$R_{1}=R_{2}=R_{3}=R_{4}=2.4, B_{1}=B_{2}=0.439, T_{12}=0.545 \mathrm{~s}$

\section{REFERENCES}

[1] Nindul Sinha, Loi Lei Lai, Venu Gopal Rao, (April 2008) "GA optimized PID controllers for automatic generation control of two area reheat thermal system under deregulated environment", proc. IEEE international conference on electric utilizes deregulation and restructuring and power technologies,6-9, pp. 1186-1191.

[2] V Donde, M.A. Pai, I.A. Hiskens, "Simulation and optimization in an AGC system after deregulation", IEEE Trans. on Power Systems., Aug. 2001, vol. 16, no. 3, pp. 481-489.

[3] O.I. Elgerd, (2001) "Electric energy system theory - an introduction", McGra- Hill Co., 2001.

[4] J. Kennedy, R. Eberhart, Y. Shi, Swarm intelligence, Morgan Kaufmann Publishers, San Francisco, 2001.

[5] M. Clerc, J. Kennedy, The particle swarm-explosion, stability, and convergence in a multidimensional complex space, IEEE Trans. On Evolutionary Computation, Vol. 6, No. 1, 2002, pp. 58-73.

[6] J. Kumar, K. Ng, and G. Sheble, "AGC simulator for price-based operation: Part I," IEEE Trans. Power Systems, vol. 12, no. 2, May 1997

[7] “AGC simulator for price-based operation: Part II," IEEE Trans. Power Systems, vol. 12, no. 2, May 1997.

[8] R. Christie and A. Bose, "Load-frequency control issues in power systems operations after deregulation," IEEE Trans. Power Systems, vol.11, pp. 1191-1200, Aug. 1996.

[9] E. Nobile, A. Bose, and K. Tomsovic, "Bilateral market for load following ancillary services," in Proc. PES Summer Power Meeting,Seattle, WA, July 15-21, 2000.

\section{BIOGRAPHIES}

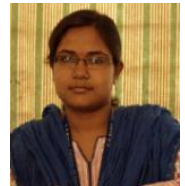

S. Rout is currently Assistant Professor in Department of Electrical Engineering, C. V Raman College of engineering, Bhubaneswar, India. She received her M.Tech in Electrical Engineering with specialization in Power System Engineering from VSSUT, Burla, India in the year 2013. Her areas of research interest are modeling of power systems, stability and control of large scale systems, particle swarm optimization and its applications.

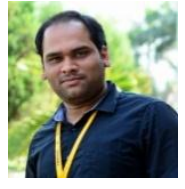

K. Panda is currently Assistant Professor in Department of Electrical Engineering, C. $\mathrm{V}$ Raman College of engineering, Bhubaneswar, India. He received his M.Tech in Electrical Engineering with specialization in Power Electronics and Drives from KIIT University, Bhubaneswar, India in the year 2013. His areas of research interest are Power electronics and drives, Power system applications, particle swarm optimization, Genetic Algorithm and its applications. 